\title{
Effectiveness of low-dose radiation therapy to improve mortality of COVID-19
}

\author{
Hamid Ghaznavi ${ }^{1}$ (])
}

Received: 7 May 2021 / Accepted: 21 June 2021 / Published online: 5 July 2021

(c) The Author(s), under exclusive licence to Springer-Verlag GmbH Germany, part of Springer Nature 2021

\begin{abstract}
Introduction Performing low-dose radiation therapy (LDRT) is a new approach to treat pneumonia resulting from COVID19 disease. This paper aims to evaluate the effectiveness of LDRT in treating COVID-19 patients.

Methods Medline was searched for "low-dose" and "radiation therapy" and "COVID-19" and "pneumonia" and "inflammation", to retrieve papers that published on low-dose radiation therapy to improve mortality of COVID-19 patients. Only clinical investigations that included original and case report papers were selected for this paper.

Results The completed clinical trials that have performed LDRT to treat COVID-19 showed that the effectiveness of LDRT in treating COVID-19 was up to $90 \%$.

Conclusion The vast majority of primary and secondary outcomes of clinical trial investigations regarding LDRT in treating COVID-19 found that LDRT can be considered a feasible treatment to improve mortality of COVID-19 patients.
\end{abstract}

Keywords COVID-19 $\cdot$ Low-dose radiation therapy $\cdot$ Pneumonia

\section{Introduction}

Coronavirus disease 2019 (COVID-19) is spreading rapidly throughout the world. Infection of people by this virus leads to pneumonia and acute respiratory distress syndrome (ARDS). COVID-19 infects the lower respiratory system, and the lung's response to this infection is recruiting macrophages and monocytes leading to inflammation, which causes widespread damage to the lung's airways (Tay et al. 2020).

Performing low-dose radiation therapy (LDRT) is a new approach to treat pneumonia resulting from COVID-19. It has been found that LDRT can lead to improvement in inflammation in different line cells, animals, and humans (Ghaznavi et al. 2021). Based on the previous clinical trials, the effectiveness of LDRT in treating COVID-19 was up to 80\%; therefore, the Food and Drug Administration (FDA) recommended LDRT (by irradiating $0.5 \mathrm{~Gy}$ ) as a treatment for COVID-19 (Hahn and Hahn 2020).

Hamid Ghaznavi

hamid.ghaznavi@muk.ac.ir

1 Department of Radiology, Faculty of Paramedical Sciences, Kurdistan University of Medical Sciences, Sanandaj, Iran
At the moment, 16 different groups throughout the world have applied LDRT to treat COVID-19 in humans, and the results of some have been published (www.clinicaltrials. gov/ct $2 /$ results? recrs $=\&$ cond $=$ Covid $19 \&$ term $=$ low + dose + radiation $\&$ cntry $=\&$ state $=\&$ city $=\&$ dist $)$. Table 1 illustrates clinical trials all around the world. These studies have categorized based on the prospective study. The first attempts to use LDRT to treat COVOD-19 were made by Ameri et al. and Khan et al. The results of these clinical studies showed that the efficacy of LDRT was noteworthy for treating inflammation and pneumonia caused by COVID-19 in human (Hess et al. 2020; Ameri et al. 2020). This study aims to evaluate the results of the outcomes of completed clinical trial in performing LDRT to treat COVID-19 patients.

Medline was searched for "low-dose [MESH]" and "radiation therapy [MESH]" and "COVID-19 [MESH]", and "pneumonia [MESH]" and "inflammation [MESH]", to retrieve papers that published on low-dose radiation therapy to improve mortality of COVID-19 patients. Papers publishing data on COVID-19 and low-dose radiation therapy between 2019 and the first of April 2021 were included in this review. Articles in English language were reviewed. References of the articles were screened for other papers and included in this review when considered relevant. 
Table 1 clinical trials in using LDRT to treat COVID-19 patients

\begin{tabular}{|c|c|c|c|}
\hline Study Title & Status & Location & $\begin{array}{l}\text { Estimated study } \\
\text { completion date }\end{array}$ \\
\hline Low-dose radiation therapy for COVID-19 pneumonia & Active, not recruiting & India & September 2020 \\
\hline $\begin{array}{l}\text { Low-dose radiation therapy for severe-acute-respiratory-syndrome-coronavirus-2 (SARS- } \\
\text { CoV-2), COVID-19 }\end{array}$ & Completed & Switzerland & April, 2021 \\
\hline $\begin{array}{l}\text { Radiation eliminates storming cytokines and unchecked edema as a } 1 \text { day treatment } \\
\text { for COVID-19 }\end{array}$ & Suspended & USA & March, 2022 \\
\hline $\begin{array}{l}\text { Best supportive care with or without low-dose whole lung radiation therapy for the treat- } \\
\text { ment of COVID-19 }\end{array}$ & Recruiting & USA & May, 2022 \\
\hline Anti-inflammatory effect of low-dose whole-lung radiation for COVID-19 pneumonia & Completed & Mexico & January, 2021 \\
\hline Low-dose pulmonary irradiation in patients with COVID-19 infection of bad prognosis & Recruiting & Spain & January, 2021 \\
\hline $\begin{array}{l}\text { Low-dose whole lung radiation therapy for patients with COVID-19 and respiratory } \\
\text { compromise }\end{array}$ & Recruiting & USA & December, 2021 \\
\hline Low-dose radiotherapy for patients with SARS-COV-2 (COVID-19) pneumonia & Recruiting & USA & December, 2022 \\
\hline Lung irradiation for COVID-19 pneumonia & Recruiting & USA & November 2020 \\
\hline Low-dose lung radiotherapy to treat COVID-19 pneumonia & Recruiting & UK & April 2021 \\
\hline Low-dose whole lung radiotherapy for older patients With COVID-19 Pneumonitis & Not yet recruiting & - & December 2021 \\
\hline COVID-19 pneumonitis low-dose lung radiotherapy (COLOR-19) & Recruiting & Italy & August, 2022 \\
\hline Low-dose radiotherapy in COVID-19 pneumonia & Active, not recruiting & Iran & December 2020 \\
\hline Low-dose radiotherapy for COVID-19 pneumonitis & Recruiting & Spain & September,2020 \\
\hline Low-dose anti-inflammatory radiotherapy for the treatment of pneumonia by COVID-19 & Recruiting & Spain & July, 2021 \\
\hline Ultra low doses of therapy with radiation applicated to COVID-19 & Recruiting & Spain & July, 2021 \\
\hline
\end{tabular}

The inclusion criteria for this paper were published papers about applying low-dose radiation therapy to treat pneumonia resulted from COVID-19. However, the exclusion criteria conducted by eliminating editorial, commentary, letter to the editor, and review articles about low-dose radiation therapy to treat COVID-19. In fact, only clinical investigations included original and case reports papers were selected for this paper.

\section{Results of clinical trials}

Hess et al. treated five patients with COVID-19 in the age range of 64-96 years. A single dose (1.5 Gy) was delivered to the whole lungs of these patients. Results showed that in the first $24 \mathrm{~h}$ after exposure, the respiratory condition of four patients was quickly improved. They recovered at an average of 1.5 days, and were discharged at an average of 12 days, and no acute radiation toxicities were reported. Blood tests and repeated imaging confirmed that the effectiveness of LDRT in treating COVID-19 is up to 80\% (Hess et al. 2020). Phase 2 of this clinical trial was carried out on 10 patients who were irradiated by $1.5 \mathrm{~Gy}$, compared to 10 control patients-blindly matched by age and morbiditysix of the patients in the control group received COVID-19 drugs. Results showed that the median time to clinical recovery (CR), median time to hospital discharge, and intubation rates were 3 vs 12 days, 12 vs 20 days, $10 \%$ vs $40 \%$ in irradiated and control groups, respectively. Overall survival rate after 4 weeks was $90 \%$ for both groups. Obtained evidence of diagnostic imaging and hematological tests showed that LDRT can be effective in treating COVID-19 patients. Preliminary results of the investigation carried out by Khan et al. showed that performing LDRT to treat COVID-19 is feasible with high potential for treating COVID-19 patients, and the results of phase 2 of their investigation proved this claim (Hess et al. 2021).

Ameri et al. initially performed LDRT to treat COVID19 on five patients with COVID-19 aged over 60 years. The whole lungs of these patients were irradiated by a singledose fraction $(0.5 \mathrm{~Gy})$. The result indicated improvement of four patients in the first few days after irradiation. They were discharged with an average of 6 days, and no radiation toxicity was observed in these patients (Ameri et al. 2020). The final clinical trial of the investigation conducted by Ameri et al. was performed on 10 patients. Five, one, and four patients were irradiated $0.5 \mathrm{~Gy}$ in single-dose fraction, 1.0 Gy in double dose fraction, and 1.0 Gy in single-dose fraction, respectively. The mean improvements in blood oxygen level at days 1 and 2 after LDRT were $2.4 \%$ and $3.6 \%$, respectively. Nine patients were treated after 1 day. Five, one, and four patients were discharged, opted out of the trial and died in the hospital, respectively. Two out of five discharged patients died at home within 3 days. Overall, the response rate (RR)_-defined as an increase in blood 
oxygen level-of these patients and clinical recovery (CR) were $63.6 \%$ and $55.5 \%$, respectively (Ameri et al. 2021).

Sanmamed et al. carried out LDRT to treat nine patients with the median age 66 years. In this study, lungs were irradiated in single-dose fraction (1 Gy), and the SatO2/ FiO2 index of these patients was evaluated. Results showed that $\mathrm{SatO} 2 / \mathrm{FiO} 2$ index significantly improved 3 days after LDRT, and the lung inflammation decreased 1 week after LDRT. Compared to patients who did not receive LDRT, the median days of hospitalization of patients who received LDRT was reduced by approximately one-fifth. Seven patients were discharged and two patients died in the hospital, the reasons for death were sepsis and severe baseline chronic obstructive pulmonary disease (Sanmamed et al. 2019).

Del Castillo et al. performed LDRT to treat a 64-yearold patient with COVID-19. The whole lungs of this patient was irradiated by a single-dose fraction (1 Gy). Three days after, the patient showed improvement in respiratory system, and inflammatory markers decreased in patient's serum. Seven days after LDRT the patient was discharged from the ICU and no radiation toxicity was observed in these patients (Castillo et al. 2020).

Sharma et al. performed LDRT to treat 10 patients with COVID-19 the age range of 38-63 years. Both lungs of this patient was irradiated by a single-dose fraction $(0.7 \mathrm{~Gy})$. All patients completed the prescribed treatment. Nine patients completed CR mostly within a period ranging from 3 to 7 days, and discharged from hospital with the range of 10-24 days after LDRT. One patient died 24 days after LDRT. No radiation toxicity was observed in these patients. Overall, RR of these patients was $90 \%$ (Sharma et al. 2020).

Outcomes of mentioned clinical trials showed that LDRT can be considered a feasible treatment with noticeably potential improvement, but Papachristofilou et al. recently have published the results of their clinical trial that challenged previous clinical trials, and claimed that using LDRT to improve COVID-19 patients has failed. They chose 22 patients with a median age of 75 years, and divided them into two groups of 11 patients. Those in the first group were irradiated by LDRT (1 Gy), and those in the second group called sham-RT group did not receive irradiation (The medical physicist closed the multileaf collimator leaves for sham-RT group). After 15 day follow-up, survival was estimated $72.7 \%$ and $63.6 \%$ for LDRT group and sham-RT group, respectively, but after 4 weeks, survival was estimated the same for both groups $(63.6 \%)$. In addition, the difference in ventilator-free days after 15 days was not observed between the two groups. Contradictory results of this clinical trial may be due to administer the therapeutic drugs such as Remdesivir-and experimental drugs such as canakinumab and conestat alfa-and only the inclusion of patients requiring mechanical ventilation (Papachristofilou et al. 2021). Mortazavi et al. have evaluated clinical trial of Papachristofilou et al. They found that due to perform unjustified dose-the doses were not within the optimal window of dose-and probably a window of opportunity during which LDRT can effectively address the pulmonary symptoms of COVID19 or other viral pneumonias, Papachristofilou et al. study failed to find effects of LDRT for improving COVID-19 (Bevelacqua et al. 2021).

\section{Looking ahead}

The vast majority of primary and secondary outcomes of clinical trial investigations regarding LDRT in treating COVID-19 show that LDRT can be considered a feasible treatment to improve these patients up to $90 \%$, besides, FDA accepted LDRT to treat pneumonia as a strong approach to reduce mortality of COVID-19 patients. There is only one investigation that has challenged LDRT to improve COVID-19 patients. The main limitations of these studies include low sample size, perform unjustified dose, and using therapeutic drugs during clinical trials (using drugs can be considered a confounding or intervening variable). The mentioned limitation affects the outcomes of performing LDRT to improve COVID-19 patients. Therefore, judging on the use of LDRT in the treatment of COVID-19 requires further studies with higher sample size and elimination of confounding or intervening variables.

Funding None.

\section{Declarations}

Conflict of interest The author declare that they have no conflict of interest.

Ethics approval and consent to participate Not applicable.

Consent for publication Not applicable.

\section{References}

Ameri A, Rahnama N, Bozorgmehr R, Mokhtari M, Farahbakhsh M, Nabavi M et al (2020) Low-dose whole-lung irradiation for COVID-19 pneumonia: short course results. Int J Radiat Oncol Biol Phys 108(5):1134-1139

Ameri A, Ameri P, Rahnama N, Mokhtari M, Sedaghat M, Hadavand $F$ et al (2021) Low-dose whole-lung irradiation for COVID-19 pneumonia: final results of a pilot study. Int J Radiat Oncol Biol Phys 109(4):859-866 
Bevelacqua J, Welsh JS, Mortazavi SMJ (2021) Comments on: "Low dose radiation therapy for severe COVID-19 pneumonia: a randomized double-blind study." Int J Radiat Oncol Biol Phys. https:// doi.org/10.1016/j.ijrobp.2021.04.025

Del Castillo R, Martinez D, Sarria GJ, Pinillos L, Garcia B, Castillo L et al (2020) Low-dose radiotherapy for COVID-19 pneumonia treatment: case report, procedure, and literature review. Strahlenther Onkol 196(12):1086-1093

Ghaznavi H, Elahimanesh F, Abdolmohammadi J, Mirzaie M, Ghaderi S (2021) Low-dose radiation therapy: a treatment for pneumonia resulting from COVID-19. J Radiother Pract. https://doi.org/10. $1017 / \mathrm{S} 1460396920001089$

Hahn SM, Hahn DD (2020) Low-dose radiotherapy, 0.5 Gy to the lungs, for COVID-19 pneumonia. https://doi.org/10.13140/RG.2. 2.27967 .33441

Hess CB, Buchwald ZS, Stokes W, Nasti TH, Switchenko JM, Weinberg BD et al (2020) Low-dose whole-lung radiation for COVID19 pneumonia: planned day 7 interim analysis of a registered clinical trial. Cancer 126(23):5109-5113

Hess CB, Nasti TH, Dhere VR, Kleber TJ, Switchenko JM, Buchwald $\mathrm{ZS}$ et al (2021) Immunomodulatory low-dose whole-lung radiation for patients with coronavirus disease 2019-related pneumonia. Int J Radiat Oncol Biol Phys 109(4):867-879

Papachristofilou A, Finazzi T, Blum A, Zehnder T, Zellweger N, Lustenberger $\mathrm{J}$ et al (2021) Low dose radiation therapy for severe
COVID-19 pneumonia: a randomized double-blind study. Int $\mathbf{J}$ Radiat Oncol Biol Phys. https://doi.org/10.1016/j.ijrobp.2021. 02.054

Sanmamed N, Alcantara P, Cerezo E, Gaztañaga M, Cabello N, Gómez $S$ et al (2021) Low-dose radiation therapy in the management of coronavirus disease 2019 (COVID-19) pneumonia (LOWRAD-Cov19): preliminary report. Int J Radiat Oncol Biol Phys 109(4):880-885

Sharma DN, Guleria R, Wig N, Mohan A, Rath GK, Subramani V et al (2020) Low dose radiation therapy for COVID-19 pneumonia: a pilot study. medRxiv. https://doi.org/10.1101/2020.11.16.20231 514

Tay MZ, Poh CM, Rénia L, MacAry PA, Ng LF (2020) The trinity of COVID-19: immunity, inflammation and intervention. Nat Rev Immunol 20(6):363-374

www.clinicaltrials. gov/ct $2 /$ results? recrs $=\&$ cond $=$ Covid $19 \&$ term $=$ low + dose + radiation $\&$ cntry $=\&$ state $=\&$ city $=\&$ dist

Publisher's Note Springer Nature remains neutral with regard to jurisdictional claims in published maps and institutional affiliations. 